\title{
Desenvolvimento de um Sistema de Navegação para Robôs Móveis Baseado em Visão Computacional
}

\author{
Alexandre de Assis Ribeiro* João Edgar Chaves Filho** \\ Renan Landau Paiva de Medeiros ${ }^{* *}$ Iury Valente de Bessa ${ }^{* *}$ \\ * Fundação Paulo Feitoza, Manaus, Amazonas, Brasil (e-mail: \\ alexandredearibeiro@gmail.com) \\ ** Universidade Federal do Amazonas, Manaus, Amazonas, Brasil \\ (e-mails: joaoedgarc@gmail.com,renanlandau@ufam.edu.bre \\ iurybessa@ufam.edu.br)
}

\begin{abstract}
Mobile robotics has applications in many different areas, such as in industries, homes and military. For the development of navigation system for mobile robotics, localization is one of the main tasks, since this parameter is necessary to assist decisions making and plan how the robot will achieve the desired points. Several approaches to this problem are used, with odometry widely applied. However, odometry often do not present good precision results when applied to indoor environments. In this context, this work presents the development of a navigation system considering localization and position control based on computer vision applied to mobile robots in indoor environments. It is presented a comparison between the location system based on odometry and the proposed system based on computer vision. The experimental results demonstrates the efficiency of the proposed localization system and compares it to an odometrybased method.

Resumo: A robótica móvel possui aplicações nas mais diversas áreas, como em indústrias, residências e até militar. Para o desenvolvimento de um sistema de navegação para sistemas robóticos, a localização é uma tarefa primordial, uma vez que este parâmetro é necessário para planejar como robô chegará aos pontos desejados e auxiliar na tomada de decisões. São utilizadas diversas abordagens para este problema, sendo a odometria amplamente aplicada. Entretanto, a odometria costuma não apresentar bons resultados relacionados à precisão quando aplicada a ambientes internos. Neste contexto, este trabalho apresenta o desenvolvimento de um sistema de navegação (englobando localização e controle de posição) baseado em visão computacional, aplicado a robôs móveis em ambientes internos. É apresentado ainda um comparativo entre o sistema de localização baseado em odometria e o sistema proposto baseado em visão computacional. Ao fim do trabalho, é demonstrada (por meio de resultados experimentais) a eficiência do sistema de localização baseado em visão computacional em comparação com a localização por odometria.
\end{abstract}

Keywords: robotics; localization; navigation; computer vision.

Palavras-chaves: robótica; localização; navegação; visão computacional.

\section{INTRODUÇÃO}

Os sistemas de navegação robóticos possuem diversas partes que desempenham tarefas intermediárias. Uma das mais importantes tarefas dos sistemas de navegação é a localização do robô no ambiente. O processo de localização consiste em determinar a posição do robô no mapa em que ele está inserido (Siegwart and Nourbakhsh, 2004). Para tanto, sensores como GPS, lasers e encoders, são utilizados para prover informações sobre a posição do robô. O uso de GPS se torna impraticável para aplicações em ambientes fechados, uma vez que receptores mais baratos apresentam precisão de alguns metros. Outras abordagens para localização estão relacionadas ao uso de mapas pré-definidos, reconhecimento de marcas espaciais com posicionamento conhecido, entre outros, os quais costumam ser inflexíveis ou requererem tempo exploratório considerável e grande custo computacional. Um método comumente usado para estimar a localização de robôs móveis é a odometria. Nesta abordagem, o odômetro estima a posição e orientação do robô por meio da medição da velocidade das rodas e, baseando-se no modelo cinemático do robô, obtémse a sua velocidade. Em seguida, a posição é calculada por meio de integração numérica. Contudo, este processo resulta no erro da localização ao longo do tempo (Bischoff et al., 2012). Outras tarefas importantes dos sistemas de navegação tratam do planejamento de caminho (planejamento da rota a ser seguida pelo robô) e planejamento de movimento, ou seja, como o robô irá se mover de um ponto a outro seguindo o caminho especificado (Engedy and Horvath, 2019). 
Neste contexto, a utilização de câmeras para localização de robôs em ambientes fechados se torna uma importante alternativa, uma vez que este tipo de abordagem garante resultados precisos, proporcionando autonomia ao robô e permitindo identificar o ambiente ao seu redor (Engedy and Horvath, 2019; Biswas and Veloso, 2012; Hirschmuller et al., 2015; Li et al., 2014). Desta forma, este trabalho propõe a aplicação de técnicas de visão computacional para o desenvolvimento de um sistema de localização para robôs móveis. Ademais, um algoritmo de controle de posição é proposto para complementar o sistema de navegação. O desempenho do sistema de localização baseado em visão computacional é comparado com o sistema de localização baseado em odometria, mostrando que o método proposto neste trabalho apresenta boa precisão e baixo custo, sendo viável para aplicações em ambientes fechados.

O restante deste trabalho é organizado da seguinte maneira: a Seção 2 apresenta o modelo cinemático do robô móvel utilizado; na Seção 3 é mostrado o conceito básico da transformada de Hough, a qual desempenha papel fundamental neste trabalho, e também são apresentados os principais conceitos acerca dos parâmetros intrínsecos e extrínsecos de câmeras. Na Seção 4, é feita a descrição do desenvolvimento do sistema de navegação proposto, o ambiente experimental e os testes elaborados. Em seguida, na Seção 5, são mostrados os resultados e a análise dos testes realizados. Por fim, a Seção 6 apresenta as principais conclusões acerca deste trabalho.

\section{MODELO CINEMÁTICO DO ROBÔ MÓVEL}

Em robótica móvel, é preciso o conhecimento do comportamento mecânico do robô, de modo que sejam utilizados robôs apropriados para as tarefas a serem realizadas, bem como desenvolver softwares de controle para o mesmo. Objetivando compreender os movimentos do robô, é necessário primeiramente descrever a contribuição das rodas para o movimento, pois cada roda possui seu papel no movimento completo do robô, como pode ser visto na obra de Siegwart and Nourbakhsh (2004).

Neste trabalho, o robô móvel omnidirecional Robotino da Festo foi utilizado. O robô possui três unidades motoras compostas cada uma por um motor CC, um encoder incremental, caixa de engrenagens e uma roda omni. Estas unidades motoras encontram-se separadas de $120^{\circ}$ entre si. Desta forma, é possível deduzir a relação cinemática entre a velocidade global do robô, $\left[\dot{x}_{g}(t) \dot{y}_{g}(t) \dot{\phi}_{g}(t)\right]^{T}$, e a velocidade das rodas, $\dot{\theta}=\left[\omega_{1}(t) \omega_{2}(t) \omega_{3}(t)\right]^{T}$, dadas por (1) e (2).

$$
\begin{gathered}
{\left[\begin{array}{l}
\dot{x}_{g}(t) \\
\dot{y}_{g}(t) \\
\dot{\phi}_{g}(t)
\end{array}\right]=r T_{r o t}^{-1} \cdot \dot{\theta}} \\
T_{\text {rot }}=\left[\begin{array}{lll}
-\sin \left(\phi_{g}(t)+\alpha_{1}\right) & \cos \left(\phi_{g}(t)+\alpha_{1}\right) & L \\
-\sin \left(\phi_{g}(t)+\alpha_{2}\right) & \cos \left(\phi_{g}(t)+\alpha_{2}\right) & L \\
-\sin \left(\phi_{g}(t)+\alpha_{3}\right) & \cos \left(\phi_{g}(t)+\alpha_{3}\right) & L
\end{array}\right]
\end{gathered}
$$

onde $r$ é o raio da roda, $L$ é a distância do centro da roda até o centro do robô e $\phi_{g}$ é a orientação angular. A odometria é baseada no movimento do robô, ou seja, utiliza a leitura dos encoders dos motores e as relações cinemáticas descritas em (1). Desta forma, o mecanismo da odometria pode estimar a posição e orientação do robô através de (3).

$$
\left[\begin{array}{l}
x_{g}\left(t_{1}\right) \\
y_{g}\left(t_{1}\right) \\
\phi_{g}\left(t_{1}\right)
\end{array}\right]=\left[\begin{array}{l}
x_{g}\left(t_{0}\right) \\
y_{g}\left(t_{0}\right) \\
\phi_{g}\left(t_{0}\right)
\end{array}\right]+\int_{t=t_{0}}^{t_{1}}\left[\begin{array}{c}
\dot{x}_{g}\left(t_{0}\right) \\
\dot{y}_{g}\left(t_{0}\right) \\
\dot{\phi}_{g}\left(t_{0}\right)
\end{array}\right] d t
$$

A cada instante a distância percorrida pelo robô é calculada através de (3), resultando na posição do robô em relação ao ponto inicial, onde o tempo de integração nada mais é do que o tempo entre as interrupções no feixe de luz do encoder incremental. Substituindo (1) em (3) e passando a relação resultante para o tempo discreto, a posição e orientação do robô pode ser calculada, resultando em (4).

$$
\left[\begin{array}{l}
x_{g}(k) \\
y_{g}(k) \\
\phi_{g}(k)
\end{array}\right]=\left[\begin{array}{l}
x_{g}(k-1) \\
y_{g}(k-1) \\
\phi_{g}(k-1)
\end{array}\right]+\Delta_{t} r \mathbf{T}_{r o t}^{-1}(k-1) \cdot \dot{\theta}(k-1)
$$

onde, $\Delta_{t}$ é o tempo de amostragem e a matriz $\mathbf{T}(k-1)$ é a matriz de transformação de coordenadas locais do robô para coordenadas globais na forma discreta.

\section{FUNDAMENTAÇÃO TEÓRICA}

\subsection{Transformada de Hough}

A transformada de Hough é uma das técnicas mais eficientes para detecção de padrões, como linhas e círculos, em imagens ruidosas. Esta técnica consiste em aplicar uma transformação nos pontos que pertencem a uma mesma curva, de tal forma que estes sejam mapeados em um único ponto em outro espaço de parametrização da curva procurada. Inicialmente era usada apenas para detecção de linhas retas em imagens binárias (veja Umbaugh (2010) e Bradski and Kaehler (2008)).

Na generalização da transformada de Hough proposta por Ballard (1981) é escolhido um ponto de referência $\left(x_{c}, y_{c}\right)$ dentro do objeto. Em seguida, é traçado um segmento de reta que une o ponto de referência a um ponto da borda, onde a direção do gradiente pode ser calculada, como pode ser visto na Figura 1.

É gerada uma tabela contendo os parâmetros $t$ e $\alpha$, como função da direção da borda no ponto de interseção, os quais são calculados por meio de (5) e (6).

$$
\begin{gathered}
t=\sqrt{\left(x-x_{c}\right)^{2}+\left(y-y_{c}\right)^{2}} \\
\alpha=\arctan \left(\frac{y-y_{c}}{x-x_{c}}\right)
\end{gathered}
$$

Vale lembrar que diferentes pontos na borda do objeto podem ter a mesma direção, fazendo com que existam mais de um par $(t, \alpha)$ para cada ângulo $\theta$. Desta forma, para detectar a forma e obter sua posição na imagem, basta buscar, para cada ponto $(x, y)$ da borda, a linha na tabela cujo ângulo $\theta$ é mais próximo à direção do gradiente no ponto $(x, y)$. Definindo uma matriz de acumulação $A$ para guardar as células do espaço de parâmetros definido como

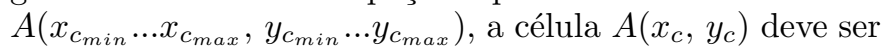


incrementada em 1. Depois de realizar este procedimento para todos os pontos da borda, são verificadas as células da matriz $A$ que excedem um determinado limiar. Estas células representam as possíveis localizações da forma do objeto na imagem.

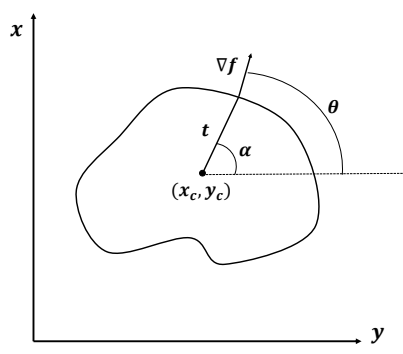

Figura 1. Geometria da transformada de Hough generalizada.

\subsection{Parâmetros Intrínsecos e Extrínsecos de Câmeras}

As lentes das câmeras costumam distorcer a localização dos pixeis nas bordas da imagem. As distorções de lentes são descritas por meio de cinco parâmetros: os coeficientes de distorção radial, $k_{1}, k_{2}$, e $k_{3}$, e os os coeficientes de distorção tangencial, $p_{1}$ e $p_{2}$. Estes parâmetros podem ser agrupados no vetor de distorção $K$ (Bradski and Kaehler, 2008).

Um ponto no plano de imagem de uma câmera pode ser representado por um vetor tridimensional $q=\left(q_{1}, q_{2}, q_{3}\right)$. Com isso, as características construtivas da câmera são representadas por meio de uma matriz $M_{3 x 3}$, chamada de matriz de parâmetros intrínsecos. A relação descrita por (7) é chamada de transformação projetiva e relaciona os pontos $Q_{i}$ no mundo físico com os pontos $q_{i}$ na tela de projeção da câmera (Dong et al., 2016).

$$
\begin{gathered}
q=M Q \\
q=\left[\begin{array}{c}
x \\
y \\
w
\end{array}\right], M=\left[\begin{array}{ccc}
f_{x} & 0 & c_{x} \\
0 & f_{y} & c_{y} \\
0 & 0 & 1
\end{array}\right], Q=\left[\begin{array}{c}
X \\
Y \\
Z
\end{array}\right]
\end{gathered}
$$

onde, os termos $c_{x}$ e $c_{y}$ representam o possível deslocamento do centro de coordenadas na tela de projeção. Já os termos $f_{x}$ e $f_{y}$ definem o comprimento focal, em pixeis (veja Dong et al. (2016) e Bradski and Kaehler (2008)).

Quando uma câmera captura a imagem de um objeto é possível descrever a posição deste objeto em relação ao sistema de coordenadas da câmera, por meio de uma rotação e translação. Matematicamente, isto é feito através da multiplicação do vetor de coordenadas do ponto por uma matriz quadrada de dimensão apropriada. Com isso, podem ser feitas rotações tridimensionais, utilizando matrizes $3 \mathrm{x} 3$ que descrevem uma rotação $\theta$ em torno do eixo $Z, \psi$ em torno do eixo $X$ e $\phi$ em torno do eixo $Y$. $\mathrm{O}$ vetor de translação representa um deslocamento de um sistema de coordenadas para outro sistema, onde a origem está em outra posição. Sendo assim, para deslocar a origem de um sistema de coordenadas centrado em um objeto para outro centrado na câmera, o vetor de translação, $T$, é simplesmente a subtração dos vetores que descrevem a posição das origens dos dois sistemas de coordenadas. Um ponto $P_{o}$ no sistema de coordenadas do objeto, possui coordenadas $P_{c}$ no sistema de coordenadas da câmera. $\mathrm{Na}$ relação descrita em (8), os termos $R$ e $T$ são definidos como os parâmetros extrínsecos da câmera.

$$
P_{c}=R\left(P_{o}-T\right)
$$

\section{SISTEMA DE NAVEGAÇÃO BASEADO EM VISÃO COMPUTACIONAL}

\subsection{Calibração da câmera}

A câmera utilizada para o desenvolvimento deste trabalho foi fixada no teto do ambiente usado para os experimentos. A Figura 2 mostra o posicionamento dos principais componentes deste trabalho. A câmera é conectada a um computador de mesa por meio de um cabo USB (Universal Serial Bus). O computador em questão é equipado com um processador Intel Core i7-4790 CPU 3,60 GHz, 16 GB de memória RAM e sistema operacional Windows 1064 bits.

O processo de calibração foi realizado por meio da toolbox Camera Calibrator presente no software MATLAB v9.3 (R2017B). Inicialmente foram capturadas 22 imagens de um padrão xadrez 7x9 em diferentes posições e carregadas para a toolbox. Finalizada a calibração, os parâmetros intrínsecos da câmera (coeficientes de distorção e comprimento focal) foram exportados em forma matricial para o workspace do MATLAB.

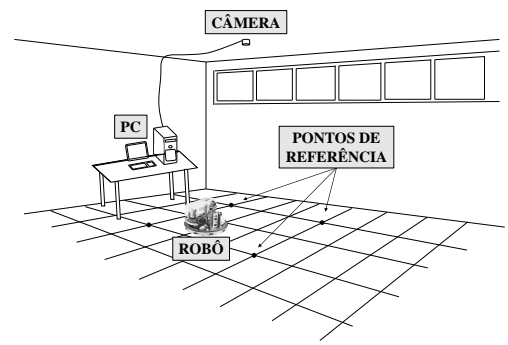

(a)

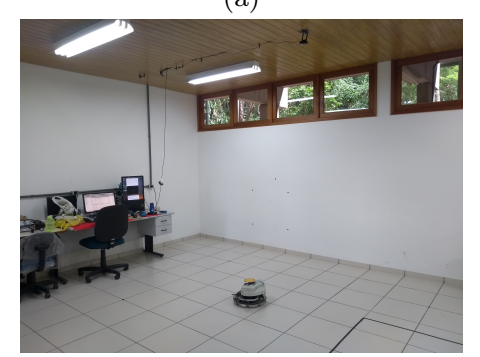

(b)

Figura 2. Montagem do trabalho desenvolvido. (a) ilustração das partes do projeto; (b) imagem real do ambiente de testes.

Para a obtenção da posição em unidade física de um ponto em uma imagem é necessário primeiramente mapear a posição de quatro pontos na imagem (chamados de pontos de imagem), com sua correspondente posição em um sistema de coordenadas pré definido (chamados de pontos do modelo). Esta etapa inicial foi feita com o auxílio de um programa em $\mathrm{C}++$, em que este captura a imagem do mapa e o mostra na tela. Ao clicar em um dos pontos 
de referência mostrados na imagem, o programa exibe a posição deste ponto em pixeis, a qual foi anotada. Para o processamento das imagens provenientes da câmera foi utilizada a biblioteca OpenCV (Open Source Computer Vision Library) na sua versão 3.4.2 para linguagem $\mathrm{C}++$.

\subsection{Deteç̧ão do robô na imagem}

Para a detecção do robô na imagem foi marcado um círculo de cor laranja sobre o Robotino, posicionado de tal forma que seu centro coincida com o centro geométrico do robô. Para diminuir o esforço computacional para a detecção do círculo, foram realizadas duas transformações na imagem. A primeira é uma transformação para o espaço de cores HSV (Hue/Saturation/Value), na qual as informação de cores são transformadas para um espaço matemático que desacopla as informações de brilho e cor, como mostrado em Davies (2012) e Umbaugh (2010). A segunda operação realizada na imagem é a binarização, onde cada pixel na imagem pode assumir os valores de preto e branco ou "0"e "1". Neste tipo de imagem apenas um dígito binário representa cada pixel. Os valores de matiz, saturação e valor (HSV) foram ajustados de tal forma que apenas o círculo laranja apareça na imagem após a binarização, como mostra a Figura 3.

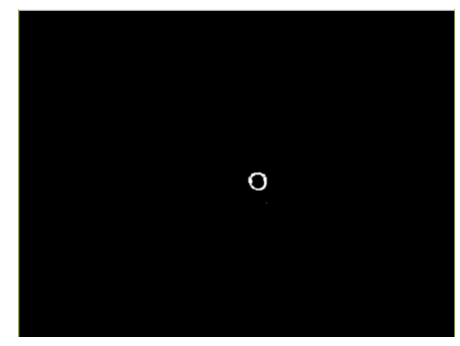

Figura 3. Imagem do mapa transformado para o espaço HSV e depois binarizada, onde aparece apenas a borda do círculo laranja.

\subsection{Posição do robô a partir do processamento de imagem}

Inicialmente, dentro do programa principal de processamento de imagem, são inseridos os valores dos parâmetros HSV e a posição dos pontos de referência obtidos anteriormente. Inicia-se então o loop principal do código, onde a próxima imagem é capturada e transformada para o espaço HSV. Após esta transformação é aplicado o algoritmo de Hough para detectar os círculos na imagem. Como mostra a Figura 3, pressupõe-se que na imagem exista apenas um círculo, ou seja, o círculo laranja colocado sobre o robô. Como resultado do algoritmo de Hough, é obtida a posição $\left(x_{c_{\text {pixel }}}, y_{c_{\text {pixel }}}\right)$ e o raio $r$ deste círculo, estando todos estes valores em pixeis. Sendo este círculo concêntrico com o centro geométrico do robô, logo obtém-se a localização do robô na imagem. Em seguida, os pontos de referência, a matriz de parâmetros intrínsecos e os coeficientes de distorção, são usados para obter a matriz de rotação e o vetor de translação. Isto é feito por meio das funções SolvePnP e Rodrigues, próprias da biblioteca OpenCV. A partir destes parâmetros é possível calcular o vetor $[X, Y, Z]^{T}$ que descreve a posição global do robô, a partir de (9), como pode ser visto em Umbaugh (2010) e Bradski and Kaehler (2008).

$$
\left[\begin{array}{l}
X \\
Y \\
Z
\end{array}\right]=R^{-1}\left(M^{-1} s\left[\begin{array}{l}
u \\
v \\
1
\end{array}\right]-T\right)
$$

onde, $R$ é a matriz de rotação, $T$ é o vetor de translação, $M$ é a matriz de parâmetros intrínsecos, $[u, v, 1]$ é a posição do ponto a ser localizado na imagem, que neste caso correspondem a $u=x_{c_{\text {pixel }}}$ e $v=y_{c_{\text {pixel }}}$ e o valor de $s$ é descrito por meio da seguinte relação:

$$
s\left[\begin{array}{l}
u \\
v \\
1
\end{array}\right]=M\left(R\left[\begin{array}{l}
X \\
Y \\
Z
\end{array}\right]+T\right)
$$

Vale ressaltar que com esta abordagem é possível obter apenas a posição do robô, e não sua orientação. Assumese, então que o sistema de referência do robô inicialmente coincide com o sistema de coordenadas do mapa onde o robô está.

\subsection{Algoritmo de controle de posição}

A Figura 4 mostra a estrutura do sistema de navegação baseado em visão computacional. A posição desejada para o robô aparece como entrada do controlador de posição executado no MATLAB. Este controlador compara a posição desejada com a posição atual do robô, obtida por meio do processamento da imagem do mapa. Em seguida, a referência de velocidade é calculada e enviada para o controlador PID, interno ao Robtino, por meio da sua API, fechando assim o ciclo de controle até que o robô atinja o ponto desejado com o erro previamente estipulado.

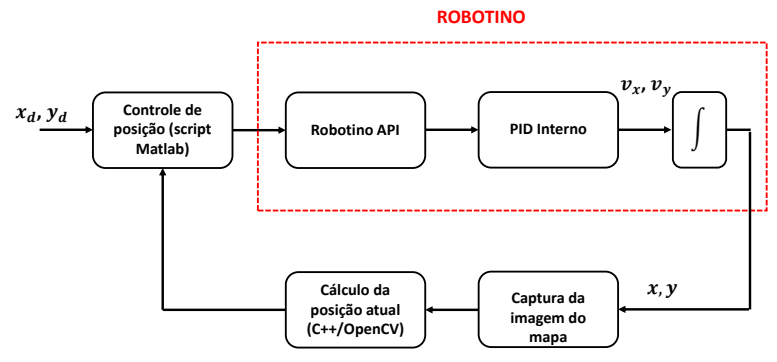

Figura 4. Diagrama em blocos do controle de posição baseado em visão computacional.

Para a validação do sistema de localização baseado em visão computacional, foi desenvolvido um script em MATLAB para o controle de posição, representado pelo Algoritmo 1. Este script recebe uma matriz $P_{n \times 2}$, onde $n$ é o número de pontos a serem visitados pelo robô. Uma vez que estes pontos são previamente conhecidos, é possível calcular o ângulo desejado por meio do arco tangente do vetor que liga o ponto atual e próximo ponto a ser alcançado e assim é calculado o ângulo $\phi_{d}$. A partir deste ângulo, pode-se calcular as componentes da velocidade resultante para o ponto desejado por meio de (11).

$$
\begin{aligned}
& v_{x}=V \cos \left(\phi_{d}\right) \\
& v_{y}=V \operatorname{sen}\left(\phi_{d}\right)
\end{aligned}
$$

onde, $V$ é o módulo da velocidade. Os valores de $v_{x}$ e $v_{y}$ formam a referência para o controlador PID que é interno 
ao Robotino, o qual calcula a corrente necessária para os motores, baseado no modelo dinâmico do robô.

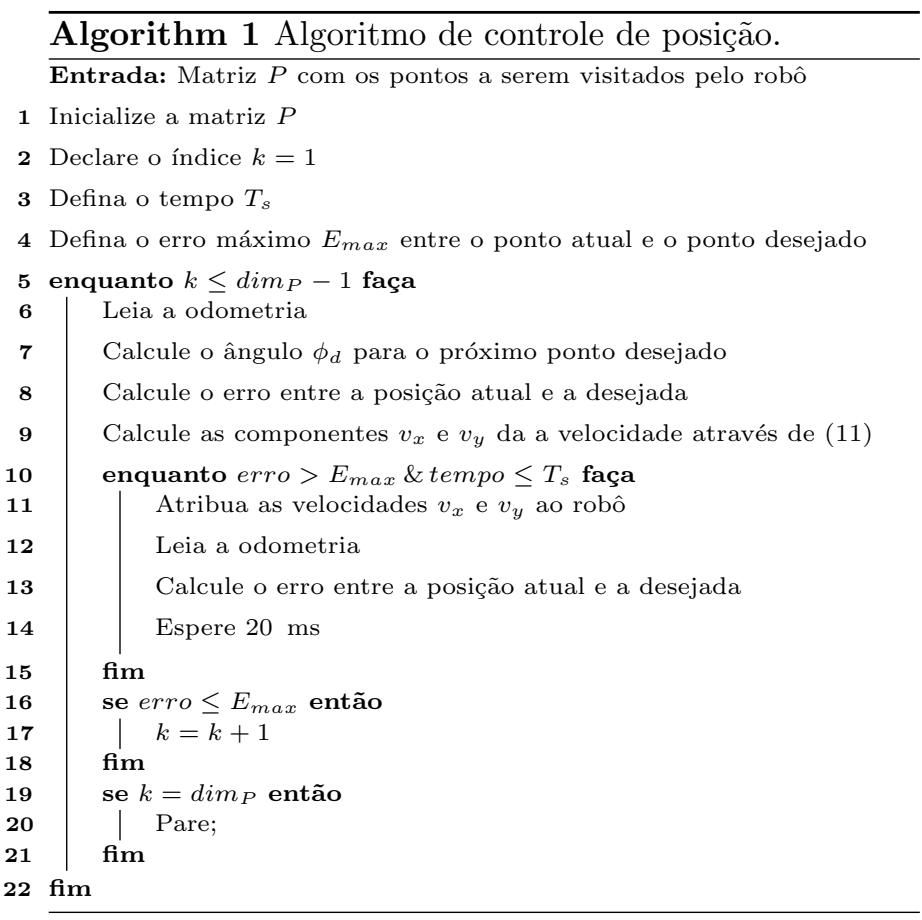

Enquanto o robô se move de um ponto a outro com as velocidades calculadas por meio de (11), o erro entre a posição atual e a posição desejada (distância euclidiana entre estes dois pontos) é calculado. Quando este erro se torna menor ou igual ao erro previamente estipulado, o índice $k$ é atualizado. Este índice controla o laço mais externo no algoritmo e define quando este chega ao seu fim, como mostra o Algoritmo 1. Já o laço mais interno é controlado pelo erro e por um tempo $T_{s}$, definido no início do algoritmo. Dentro deste laço, são atribuídas as velocidades $v_{x}$ e $v_{y}$ ao robô e é calculado o erro entre a posição atual e a desejada. O tempo $T_{s}$ é necessário para que um novo ângulo $\phi_{d}$ seja calculado, quando o tempo transcorrido é maior que $T_{s}$. A cada iteração do índice $k$ o robô se move de um ponto a outro da matriz $P$, até que o robô atinja o alvo (ponto final).

\section{AVALIAÇÃO EXPERIMENTAL}

\subsection{Resultados}

Como resultado da calibração foram retornados os parâmetros intrínsecos da câmera, bem como os coeficientes de distorção radial e tangencial, os quais estão dispostos abaixo, onde $\mathbf{K}$ é o vetor de coeficientes de distorção.

$$
\begin{gathered}
M=\left[\begin{array}{ccc}
630,5 & 0 & 261,8 \\
0 & 657,2 & 289,7 \\
0 & 0 & 1
\end{array}\right] \\
K=[0,1731-1,9305-0,02470,03214,0117]
\end{gathered}
$$

Conforme descrito na Seção 4, um programa escrito em $\mathrm{C}++$ foi responsável pelo cálculo da posição dos pontos de referência, tanto em milímetros quanto em pixeis. Após este processo, foi utilizada a função cv: :Point () da biblioteca OpenCV, para projetar os pontos de referência sobre a imagem, como mostra a Figura 5.
A partir da Figura 5 também percebe-se que a aplicação da transformada de Hough se mostrou efetiva, identificando corretamente o círculo laranja marcado sobre o robô. Após a identificação do círculo, a sua borda é destacada na imagem (em vermelho) bem como o centro (em verde).

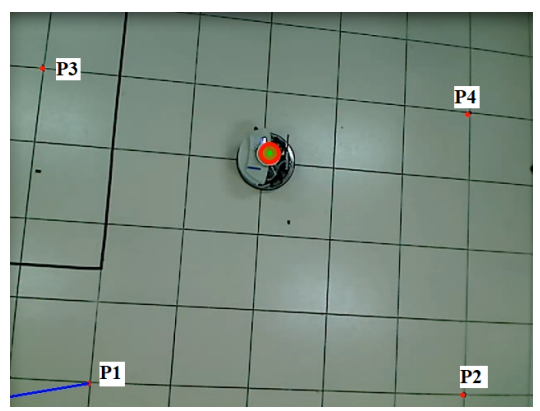

Figura 5. Projeção dos pontos de referência sobre a imagem do mapa. Vê-se também a identificação do círculo laranja sobre o robô.

Descrição dos Testes Realizados Para avaliar o desempenho do controlador de posição foram definidas duas trajetórias, a serem seguidas pelo robô: uma circular e outra retangular. Além disso, o parâmetro erro máximo foi definido como $E_{\max }=0,025 \mathrm{~m}$ e o tempo $T_{s}=0,1 \mathrm{~s}$. Para a trajetória circular, a matriz $P$ com os pontos a serem seguidos possui dimensão $41 \times 2$. Estes pontos descrevem uma circunferência centrada em $\left(x_{c}, y_{c}\right)=(1 \mathrm{~m}, 1 \mathrm{~m})$ e raio $r=0,55 \mathrm{~m}$. Após a inserção da matriz $P$ no controlador de posição, foi dado início à execução do programa que calcula a posição do robô utilizando a biblioteca OpenCV. Em seguida foi executado o script que implementa o controle de posição.

Quando o robô completa a sua trajetória são plotados os gráficos que representam a localização dele baseada em visão computacional, a trajetória ideal que deveria ser seguida e a trajetória seguida a partir da localização pelo odômetro próprio do Robotino, como se pode ver na Figura 6. O segundo teste realizado levou em conta uma trajetória retangular, onde a matriz $P$ possui dimensão $2 \times 40$. O procedimento descrito anteriormente para a trajetória circular foi repetido. Os resultados deste teste são mostrados na Figura 7. A Tabela 1 mostra o erro médio associado aos dois testes de validação realizados.

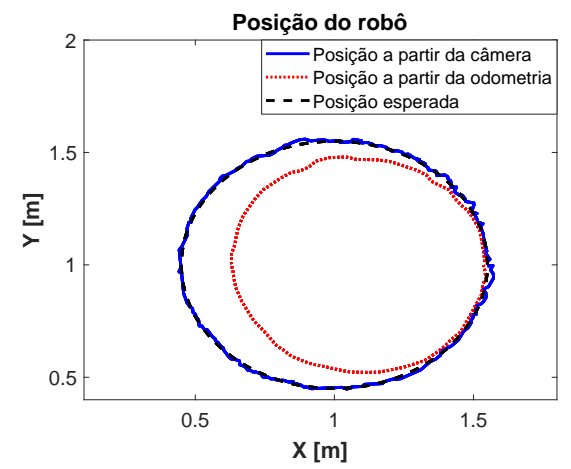

Figura 6. Trajetória a ser seguida pelo robô e trajetórias descritas a partir da localização por odometria e visão computacional para o teste com trajetória circular. 


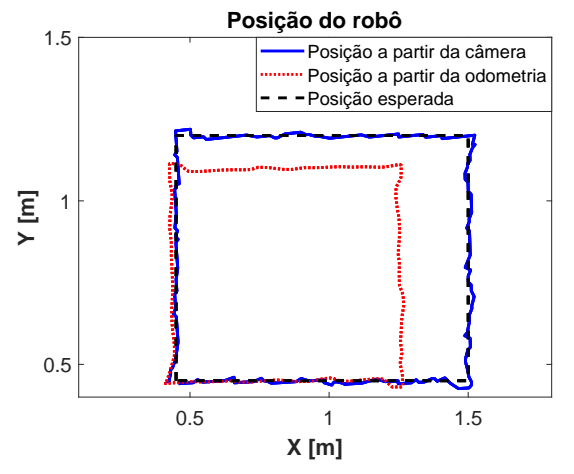

Figura 7. Trajetória a ser seguida pelo robô e trajetórias descritas a partir da localização por odometria e visão computacional para o teste com trajetória retangular.

Tabela 1. Coordenadas dos pontos de referência utilizados para a localização do robô.

\begin{tabular}{lll} 
Trajetória & $\begin{array}{l}\text { Erro } \\
\text { médio da } \\
\text { localização } \\
\text { por câmera } \\
{[\mathbf{m}]}\end{array}$ & $\begin{array}{l}\text { Erro } \\
\text { da } \\
\text { zação } \\
\text { odometria } \\
{[\mathbf{m}]}\end{array}$ \\
\hline Retangular & 0,0235 & $\begin{array}{r}\text { médio } \\
\text { pocali- }\end{array}$ \\
Circular & 0,0168 & 0,1477 \\
\hline
\end{tabular}

\subsection{Discussões}

A partir das Figuras 6 e 7, verifica-se que a posição do robô estimada pelo odômetro, apresenta diferença significativa em relação à posição dada pela localização por visão computacional, a qual está mais próxima da posição desejada. Isto já era esperado, tendo em vista que o odômetro realiza cálculos integrativos para obter a posição do robô a partir da sua velocidade. Além disso, condições ambientais, como piso escorregadio e inclinado, podem interferir na leitura dos encoders e, consequentemente, na posição estimada pelo odômetro.

A partir das Figuras 6 e 7 e os resultados contidos na Tabela 1, percebe-se que o sistema de navegação desenvolvido possui ótimo desempenho, garantindo que o robô concluísse a trajetória estipulada com erro médio na localização abaixo do valor máximo estipulado de $0,03 \mathrm{~m}$. Percebe-se também que o erro na trajetória retangular, tanto para a localização por visão computacional quanto para localização por odometria, é maior que o erro para trajetória circular. Isso se deve às mudanças bruscas na direção do vetor velocidade do robô, que ocorrem nos vértices do retângulo que descreve a trajetória. Percebese que os resultados apresentados possuem precisão semelhante ao desenvolvido por Baatar et al. (2014), onde um sistema de visão foi utilizado para controlar rotas pré definidas para o Robotino. Além disso, o erro médio na localização no presente trabalho foi menor que o erro médio no experimentos descritos em Bischoff et al. (2012), onde o menor erro para o sistema de visão foi de $4,33 \mathrm{~cm}$.

\section{CONCLUSÃO}

O trabalho desenvolvido contribuiu para a validação de um método de localização de robôs móveis baseado em visão computacional. Além disso, foi desenvolvido um controlador de posição para a plataforma robótica Robotino, resultando em um completo sistema de navegação, o qual foi testado em experimentos reais e comparado com o tradicional método de localização por odometria. Algumas limitações do sistema de navegação desenvolvido estão relacionadas à necessidade de se ter um ambiente bem iluminado, para que a função que implementa a transformada de Hough consiga detectar de forma correta o círculo marcado sobre o robô. Além disso, o ambiente de trabalho do robô fica reduzido à área em que a câmera consegue capturar a imagem.

Entretanto, verifica-se que o sistema de navegação baseado em visão computacional obteve bons resultados, em comparação com a abordagem tradicional baseada em odometria. Além disso, a técnica desenvolvida é de baixo custo e pode ser facilmente implementada para aplicações em ambiente fechados.

\section{REFERÊNCIAS}

Baatar, G., Eichhorn, M., and Ament, C. (2014). Precise indoor localization of multiple mobile robots with adaptive sensor fusion using odometry and vision data. volume 19. doi:10.3182/20140824-6-ZA-1003.02345.

Ballard, D. (1981). Generalizing the hough transform to detect arbitrary shapes. Pattern Recognition, 13(2), 111 - 122. doi:https://doi.org/10.1016/0031-3203(81) 90009-1. URL http://www. sciencedirect.com/ science/article/pii/0031320381900091.

Bischoff, B., Nguyen-Tuong, D., Streichert, F., Ewert, M., and Knoll, A. (2012). Fusing vision and odometry for accurate indoor robot localization. In 2012 12th International Conference on Control Automation Robotics Vision (ICARCV), 347-352. doi:10.1109/ICARCV. 2012.6485183.

Biswas, J. and Veloso, M. (2012). Depth camera based indoor mobile robot localization and navigation. In 2012 IEEE International Conference on Robotics and Automation, 1697-1702. doi:10.1109/ICRA.2012.6224766.

Bradski, G. and Kaehler, A. (2008). Learning OpenCV. O'Reilly Media, Sebastopol, CA.

Davies, E.R. (2012). Computer and machine vision. Academic Press, Oxford, UK.

Dong, Y., Ye, X., and He, X. (2016). A novel camera calibration method combined with calibration toolbox and genetic algorithm. In 2016 IEEE 11th Conference on Industrial Electronics and Applications (ICIEA), 1416-1420. doi:10.1109/ICIEA.2016.7603807.

Engedy, I. and Horvath, G. (2019). A global, camera-based mobile robot localization.

Hirschmuller, H., Schmid, K., and Suppa, M. (2015). Computer vision for mobile robot navigation.

Li, I.H., Chen, M.C., Wang, W.J., Su, S.F., and Lai, T.W. (2014). Mobile robot self-localization system using single webcam distance measurement technology in indoor environments. Sensors (Basel, Switzerland), 14, 2089-109. doi:10.3390/s140202089.

Siegwart, R. and Nourbakhsh, I.R. (2004). Introduction to autonomous mobile robots, volume 1. MIT Press, Cambridge, Massachusetts, 1 edition.

Umbaugh, S.E. (2010). Digital image processing and analysis. CRC Press, New York. 